\title{
SUBSONIC POTENTIAL FLOW PAST A CIRCLE AND THE TRANSONIC CONTROVERSY
}

\author{
M. D. VAN DYKE' and A. J. GUTTMANN ${ }^{2}$
}

(Received 21 January 1981; revised 19 March 1982)

\begin{abstract}
The Mach-number senes expansion of the potential function for the two-dimensional flow of an inviscid, compressible, perfect, diatomic gas past a circular cylinder is obtained to 29 terms. Analysis of this expansion allows the critical Mach number, at which flow first becomes locally sonic, to be estimated as $M_{*}=0.39823780 \pm 0.00000001$. Analysis also permits the following estimate of the radius of convergence of the series for the maximum velocity to be made: $M_{c}=0.402667605 \pm 0.00000005$, though we have been unable to determine the nature of the singularity of $M=M_{c}$. Since $M_{c}$ exceeds $M_{*}$ by some $1.1 \%$, it follows that this particular "airfoil" can possess a continuous range of shock-free potential flows above the critical Mach number. This result hopefully resolves a 70 -year old controversy.
\end{abstract}

\section{Introduction}

The Janzen-Rayleigh approximation to subsonic flow about a right circular cylinder is used to shed light on the transonic controversy, which may be formulated as the following question: Can an airfoil have a continuous range of smooth shock-free potential flows above the critical Mach number? It has been known since the 1940's that isolated shock-free flows can exist; the question is whether they have neighbouring solutions. Morawetz [15] has shown that a smooth solution ceases to exist if the shape of the profile is varied in the supersonic region with the free-stream Mach number kept fixed. It was conjectured in the 1950's that likewise the flow breaks down for a fixed profile if the Mach number is varied, but that has never been proved. There is experimental and numerical evidence on both sides. For example, Nocilla et al. [16] have

\footnotetext{
'Department of Mechanical Engineering, Stanford University, Stanford, California 94305, U.S.A.

${ }^{2}$ Department of Mathematics, University of Newcastle, Newcastle, N.S.W. 2308.

(c) Copyright Australian Mathematical Society 1983
} 
numerical results for flow past biconvex airfoils which suggest that shock-free flows can exist for a range of values up to about $5 \%$ greater than the critical Mach number. However there remains the possibility of a pathological singularity that is not manifest until the shock wave is well developed. We expand on this point subsequently.

For the case studied here, that of steady, two-dimensional flow of an inviscid, compressible, perfect gas past a circular cylinder without circulation, no answer that is even moderately convincing has previously been given, despite numerous attempts over the past 70 years. Prior to discussing these attempts, we shall formulate the problem precisely. The cylinder is set in a uniform, oncoming stream. Following both Rayleigh [18] and Janzen [11], we have used the velocity potential as the dependent variable, rather than the stream function, as the simplification of the surface boundary condition achieved by using the stream function as the dependent variable is bought at the price of a more complicated differential equation. For similar reasons, we have eschewed the use of the seductively elegant properties of a complex-variable formulation, as this elegance is found to be obtained at the price of increased computational complexity and decreased precision.

The equation for the velocity potential $\phi$ is given by Oswatitsch [17] in Cartesian co-ordinates, and re-expressing this equation in the more appropriate polar-coordinates, we have

$$
\begin{aligned}
\phi_{r r}+\phi_{r} / r+ & \phi_{\theta \theta} / r^{2} \\
=M^{2}[ & \phi_{r}^{2} \phi_{r r}+2 \phi_{r} \phi_{\theta} \phi_{r \theta} / r^{2}+\phi_{\theta}^{2} \phi_{\theta \theta} / r^{4}-\phi_{r} \phi_{\theta}^{2} / r^{3} \\
& \left.+(\gamma-1)\left(\phi_{r}^{2}+\phi_{\theta}^{2} / r^{2}-1\right)\left(\phi_{r r}+\phi_{r} / r+\phi_{\theta \theta} / r^{2}\right) / 2\right],
\end{aligned}
$$

where the variables have been made dimensionless by scaling lengths by the cylinder radius $a$ and velocities by the uniform-stream speed $U$. In the above equation, $M$ is the free-stream Mach number and $\gamma$ is the adiabatic ratio. The appropriate boundary conditions are

$$
\begin{gathered}
\phi_{r}=0 \quad \text { at } r=1, \\
\phi \sim r \cos \theta \quad \text { as } r \rightarrow \infty,
\end{gathered}
$$

together with the requirement that $\phi$ be an even function of $\theta$, to ensure symmetric flow, free of circulation. For $M=0$ the solution is

$$
\phi_{1}=(r+1 / r) \cos \theta .
$$

Frankl and Keldysh [4] have proved that $\phi\left(M^{2}\right)$ is analytic in the neighbourhood of the origin, so that $\phi$ can be written

$$
\phi=\sum_{n=1}^{\infty} \phi_{n} M^{2 n-2} .
$$


From boundary condition (2) it follows that the dimensionless surface speed $q_{s}$ is given by $-(1 / r) \partial \phi /\left.\partial \theta\right|_{r=1}$, and so $q_{s}$ can also be written as an expansion in $M^{2}$. We are interested in the maximum surface speed, which undoubtedly occurs at the ends of the diameter perpendicular to the direction of flow. Thus

$$
q_{\max }=q_{s}(\theta=\pi / 2)=\sum_{n=1}^{\infty} q_{n} M^{2 n-2} .
$$

The analyticity of $q_{\max }$ near $M=0$ follows immediately from the analyticity of $\phi$. We denote the radius of convergence of the series (6) by $M_{c}^{2}$, and wish to compare $M_{c}$ with the critical Mach number $M_{*}$, the free-stream Mach number at which flow locally becomes sonic for the first time. The critical Mach number $M_{*}$ is given by the solution of

$$
M_{*}^{2}=2 /\left[(\gamma+1) q_{\max }^{2}-\gamma+1\right] .
$$

The fundamental question we wish to resolve is whether (a) $M_{*}<M_{c}$, or (b) $M_{*}=M_{c}$. If the first possibility were true, the transonic controversy posed in the opening paragraph would be answered in the affirmative. If the second possibility were true, the position and nature of the singularity in the complex plane would need to be investigated before comment could be made on the transonic controversy. We subsequently show that the first possibility appears to hold.

Referring now to earlier studies of this problem, the first attempt was made by O. Janzen [11] in 1913 and by Lord Rayleigh [18] in 1916. Both independently obtained $\phi_{1}$ and $\phi_{2}$, and Lord Rayleigh expressed the view that $M_{c}=M_{*}$. In 1928 Taylor and Sharman [25] supported Rayleigh's view for the cylinder, but pointed out that there was "no theoretical ground for supposing that this hypothesis be true in general". Sauer [20] in 1943 also supported Rayleigh's view, but in 1960 [21] he retracted this statement, preferring to leave the question open. Ward [28] in 1953 put forward an opposing view to that of Lord Rayleigh, while a more complex view was advanced by Shapiro [23] who nevertheless agreed that $M_{*}=M_{c}$. For both the cylinder and the sphere Lighthill [13] expressed the view that $M_{c}$ could be expected to be somewhat greater than $M_{*}$. These later conclusions presumably relied on the work of Imai [10] and Simasaki [24]. In 1938 Imai extended the series by obtaining the $\phi_{3}$ term, and in 1941 the $\phi_{4}$ term. $^{\dagger}$ In 1955 Simasaki published the next two terms $\phi_{5}$ and $\phi_{6}$, and estimated $M_{*} \approx 0.40$ and

\footnotetext{
${ }^{\dagger}$ Footnote added in proof: In 1952 Max Munk and George Rawling ("Calculation of compressible subsonic flow past a circular cylinder", NA VORD Rept. 2477, U.S. Naval Ordnance Lab., White Oak, $M d .$, 1952), in a pioneering application of the computer, wired the control panel of the IBM 604 Calculating Punch to calculate successive approximations and computed $\phi_{5}$ in eight hours handling and machine time. We are indebted to Andrew Van Tuyl for drawing our attention to this work, and supplying us with a copy.
} 
$M_{c} \approx 0.50$ for $\gamma=1.405$. This was a remarkable hand calculation, though as we shall see the estimate of $M_{c}$ is significantly in error. Simasaki's calculation represented the end of the classical era, and in $1970 \mathrm{G}$. Hoffman [8, 9] used an IBM $360 / 67$ to calculate the first seven terms of the expansion. Analysis for the case $\gamma=7 / 5$ gave a value of $M_{*}=0.3983 \pm 0.0002$, which compares well with the estimate $M_{*}=0.39853 \pm 0.00002$ obtained by Melnick and Ives [14] using a numcrical integration tochniquic. Hóffman âtiempted tó estimate $M_{c}$, ânu coñcluded that it lay within $5 \frac{1}{2} \%$ of $M_{*}$, which, as he points out, is far closer than Simasaki's estimate. Following Hoffman, W. C. Reynolds (unpublished) obtained two further terms, but no attempt at analysis was undertaken.

In 1977 one of us (M. V. D.) reformulated the algebraic manipulations in such a manner as to significantly increase the accuracy and efficiency of the computer program, and as a result obtained six further terms, extending the series to $\phi_{15}$. Attempts at analysis were still inconclusive, suggesting that further terms were required. We therefore obtained two additional terms in 1978. A preliminary analysis of the 17-term series [27] suggested that $M_{c}>M_{*}$ by about $4 \%$, but by no means could we rule out the possibility of equality. Attempting to resolve this problem, we refined the existing Fortran code in order to achieve optimal efficiency, and subsequently obtained two further coefficients. Subsequently L. Schwartz (private communication) pointed out that the computational complexity could be reduced from $O\left(n^{9}\right)$ to $O\left(n^{6}\right)$ by treating the triple products in (1) as repeated double products, and this was found to give a substantial improvement in both efficiency and accuracy. In this way we have obtained 24 terms in IBM

TABLE 1. Coefficients of the series for maximum speed, $q_{\max }\left(M^{2}\right) / U=\sum_{n=1}^{\infty} q_{n} M^{2 n-2}$, with $\gamma=7 / 5$

\begin{tabular}{|c|l|l|l|}
\hline$n$ & \multicolumn{1}{|c|}{$q_{n}$} & $n$ & \multicolumn{1}{|c|}{$q_{n}$} \\
\hline 1 & 2.00000000000000 & 16 & $4.26997505742413 \times 10^{8}$ \\
2 & 1.166666666666667 & 17 & $2.16247145908896 \times 10^{9}$ \\
3 & 2.57833333333333 & 18 & $1.10463770836747 \times 10^{10}$ \\
4 & 7.51464814814814 & 19 & $5.68649851009948 \times 10^{10}$ \\
5 & $2.55904074326026 \times 10$ & 20 & $2.94779009123257 \times 10^{11}$ \\
6 & $9.62632913824853 \times 10$ & 21 & $1.53777835504235 \times 10^{12}$ \\
7 & $3.87923452464374 \times 10^{2}$ & 22 & $8.06853184029690 \times 10^{12}$ \\
8 & $1.64399333300068 \times 10^{3}$ & 23 & $4.25587560801023 \times 10^{13}$ \\
9 & $7.23987123576189 \times 10^{3}$ & 24 & $2.25576351728074 \times 10^{14}$ \\
10 & $3.28626654734814 \times 10^{4}$ & 25 & $1.2010117 \times 10^{15}$ \\
11 & $1.52864040612112 \times 10^{5}$ & 26 & $6.4210705 \times 10^{15}$ \\
12 & $7.25593930837266 \times 10^{5}$ & 27 & $3.446256 \times 10^{16}$ \\
13 & $3.50326365998740 \times 10^{6}$ & 28 & $1.856327 \times 10^{17}$ \\
14 & $1.71617906236987 \times 10^{7}$ & 29 & $1.00329 \times 10^{18}$ \\
15 & $8.51351821010245 \times 10^{7}$ & & \\
\hline
\end{tabular}


quadruple precision, and 29 terms in IBM double precision. By varying the degree of precision from single to double to quadruple we can estimate the number of significant figures in the coefficients. In Table 1 we give the first 29 coefficients for the expansion $q_{\max }$ with $\gamma=7 / 5$. The coefficients are expected to be accurate to at least the number of digits quoted.

Analysis of this series gives the estimates $M_{*}=0.39823780 \pm 0.0000001$ and $M_{c}=0.402667605 \pm 0.000000005$, so that $M_{c}$ is about $1.11 \%$ larger than $M_{*}$, and the transonic controversy in the form posed in the opening paragraph appears to be resolved in the affirmative for the circular "airfoil". We emphasize that the errors quoted above are confidence limits, estimated from the apparent rate of convergence of the sequences used to estimate $M_{*}$ and $M_{c}$ and hence are not rigorous bounds.

In the next section we discuss the derivation of the series, and in the subsequent section the analysis is presented. The final section comprises a discussion and conclusion.

\section{Series derivation}

The differential equation (1) is solved by successive approximations, assuming that the free-stream Mach number is small. Writing the solution as $\phi=\phi_{1}+$ $M^{2} \phi_{2}+M^{4} \phi_{3}+\cdots$, substituting and equating like powers of $M^{2}$, and using $\phi_{1}=(r+1 / r) \cos \theta$, we have for $\phi_{2}$ the equation

$$
\nabla^{2} \phi_{2}=\left(\frac{2}{r^{7}}-\frac{4}{r^{5}}\right) \cos \theta+\frac{2}{r^{3}} \cos 3 \theta .
$$

The identity

$$
\nabla^{2} r^{n} \cos k \theta=\left(n^{2}-k^{2}\right) r^{n-2} \cos k \theta
$$

gives the particular integral of (8) vanishing at infinity,

$$
\left(\frac{1}{12 r^{5}}-\frac{1}{2 r^{3}}\right) \cos \theta-\frac{\cos 3 \theta}{4 r} \text {. }
$$

Adding multiples of the homogeneous solution that also vanish at infinity in order to satisfy boundary condition (2) then yields

$$
\phi_{2}=\left(\frac{13}{12 r}-\frac{1}{2 r^{3}}+\frac{1}{12 r^{5}}\right) \cos \theta+\left(\frac{1}{12 r^{3}}-\frac{1}{4 r}\right) \cos 3 \theta .
$$

This procedure is then automated and carried out order by order, with $\gamma$ fixed in order to obtain as many terms as possible. Careful packing of arrays is necessary in order to make the program fit on a large virtual storage machine. Optimising 
the Fortran code gave a program with complexity $O\left(n^{6}\right)$, and in $6 \frac{1}{2}$ minutes on an IBM 370/67 computer 29 double-precision coefficients were generated. In the same length of time 24 quadruple-precision coefficients were generated, using 754 kilobytes of storage. At this stage, storage is limiting the quadruple-precision calculations, while accuracy limits the double-precision calculations. As we show in the next section, the series now appears to be of adequate length to enable $M_{*}$ and $M_{c}$ to be estimated.

\section{Analysis of series}

We first consider the determination of the critical Mach number $M_{*}$. Rewriting the defining equation (7) as

$$
(\gamma+1) M_{*}^{2} q_{\max }^{2}-M_{*}^{2}(\gamma-1)-2=0,
$$

TABLE 2. Shanks table applied to sequence of estimates of $M_{*}$

$0 .+66252404$

$0.4225 \% 7127$

0.410310037

0.405246403

0.402706318

0.401269864

0.400388602

0.399815135

0.399424951

0.399150050

0.398950865

0.398803183

0.398691560

0.398605802

0.395538984

0.398486285

0.398444273

0.398410462

0.398383020

0.398360578

0.398342099

0.398326787

0.398314028

0.398303340

0.398230183

0.398216819

0.398162984

0.398114266

0.398071914
0.405518882

0.401686944

0.400149592

0.399400216

0.398989765

0.398746682

0.398594303

0.398494525

0.398426883

0.398379706

0.398346015

0.398321466

0.398303267

0.398289572

0.398279128

0. 398271070

0.398264787

0.398259842

0.398255917

0.398252777

0.398250247

0.398248195

0 398315856

0.398213832

0.398234596

0.397650506

0.397790157
0.399119580

0.398657551

0.398492693

0.395393630

0.398538314

0.398505260

0.398284501

0.398270956

0.398261842

0.398255550

0.398251110

0.398247918

0.398245585

0. 398243854

0.398242554

0.398241565

0. 398240806

0.398240216

0.398239755

0.395239392

0. 398250186

0.398275175

0.398231085

$0.39821+545$

0.397763211
0.598532599

0.398291190

0.398268368

0.398256181

0.398249456

0.398245523

0.398243092

0.398241521

0.398240472

0.398239751

0.398239246

0.398238885

0.398238622

0.3932 .38431

0.398238286

0.398238179

0.398238098

$0: 398238033$

0.398239744

0398231182

0.398259226

0.398204616

0.398231714
0.398240346

0.398242214

0.398241178

0.398239982

0 398239156

0.398238655

0.395238357

0.398238175

0.398238055

0.398237985

0.398237925

0.398257909

0.395237847

0.398237867

0.398257851

0.398237758

0.398238096

0.398238518

0.398237741

0398240697

0.398222727
0.398241548

0.398248901

0.398237319

0.398237877

0.398237922

0.398237889

0.398237824

0.398237886

0.398237565

0.398237003

0.398237930

0.398237862

0.398237858

0.398237871

0 398237831

0.398238745

0.398238157

0.398238224

0.398238159
0.398244403

0.398237851

0.398237926

0.398237903

0.398257958

0.398237856

0395237654

0.398237729

0.39623793 .5

0.398257911

0.395237858

0.398237861

0.398237861

0.398237869

0. 398238387

0.398238217

0.398238191
0.398237925

0.398237909

0. 398257919

0.398237922

0.398237828

0.398237861

0.398237799

0.398237913

0.3952379 .15

0.599237961

0 j9\$237862

0.398257861

0.398257861

0. 395238259

0.393258156
0.398237915 0.398237923 0.398237919 0398237853 0.398237840 0.398237839 0.398237964 0.393257923 $0.39823 ? 86$ ? 0.395237861 0.395237861 0. $30 \$ 237561$ 0.398238197
0.398237921 0.398237924 0.398237837 0.398237839 0.398237840 0.398237933 0.398238047 0.398237861 0.398237859 0.398237861 0.398237861
0.398237921 0398237839 0.398237841 0.398237839 0.398237403 0.398237976 0.398237859 0.398237860 0.398237862
0.398237841 0.398237840 0.398237841 0.398237651 0.398257879 0.3982 .57860 0.398237858
0.398237842 0398237840 0.398237755 0.398237561 0.398257858
0.398237842 $0.398237802 \quad 0.398237825$ 0.398237858 
and substituting the expansion $q_{\max }=\sum q_{n} M_{*}^{2 n-2}$ into (11), we obtain a sequence of polynomials whose smallest positive root gives an estimate of $M_{*}^{2}$. The square roots of this sequence of estimates are shown in the first column of Table 2 . In subsequent columns we show the result of applying a Shanks transformation [22] to the first column in order to speed the convergence. It can be seen that the method converges fairly rapidly, and allows us to make the estimate

$$
M_{*}=0.3982378 \pm 0.0000004 \text {, }
$$

where the quoted error is based on the apparent degree of convergence. We subsequently increase the precision of this estimate.

We now estimate $M_{c}$, the radius of convergence of the series $\sum q_{n} M^{2 n-2}$. A wide variety of numerical methods exist for this problem, and many of them are reviewed in Gaunt and Guttmann [5]. Most existing methods are based on the assumption that the singularity defining the radius of convergence is a simple algebraic or algebraico-logarithmic singularity. That being the case, the ratios of successive terms of the series should approach the radius of convergence in the following manner:

$$
r_{n}=q_{n} / q_{n-1}=M_{c}^{-2}\left(1+a_{1} / n+a_{2} / n^{2}+a_{3} / n^{3}+\cdots\right) .
$$

TABLE 3. Neville table extrapolation of ratios of $q_{\max }\left(M^{2}\right) / U$. The table shows values of $1 / M_{c}^{2}(n, k)$ as functions of $n$ and $k$ where $M_{c}^{2}(n, k)=\left(1+a_{1} / n+a_{2} / n^{2}+\cdots+a_{k} / n^{k}\right) / r_{n}$, where $r_{n}=$ $a_{n} / a_{n-1}$ is the ratio of successive coefficients.

\begin{tabular}{|r|ccccccc|}
\hline$n$ & $k=0$ & 1 & 2 & 3 & 4 & 5 & 6 \\
\hline 1 & 0.583333 & & & & & & \\
2 & 2.210000 & 3.836667 & & & & & \\
3 & 2.914537 & 4.323611 & 4.567084 & & & & \\
4 & 3.405403 & 4.878002 & 5.452592 & 5.720829 & & & \\
5 & 3.761694 & 5.186859 & 5.650144 & 5.795312 & 5.813933 & & \\
6 & 4.029817 & 5.370430 & 5.737574 & 5.825004 & 5.839850 & 5.845033 & \\
7 & 4.237932 & 5.486624 & 5.777107 & 5.829819 & 5.833430 & 5.830862 & 5.828500 \\
8 & 4.403832 & 5.565134 & 5.800663 & 5.839922 & 5.850025 & 5.859982 & 5.869689 \\
9 & 4.539123 & 5.621446 & 5.818541 & 5.854298 & 5.872267 & 5.890061 & 5.905100 \\
10 & 4.651602 & 5.663914 & 5.833785 & 5.869347 & 5.891921 & 5.911575 & 5.925917 \\
11 & 4.746662 & 5.697262 & 5.847328 & 5.883448 & 5.908125 & 5.927570 & 5.940900 \\
12 & 4.828133 & 5.724308 & 5.859542 & 5.896183 & 5.921653 & 5.940592 & 5.935613 \\
13 & 4898801 & 5.746819 & 5.870629 & 5.907586 & 5.933243 & 5.951788 & 5.964849 \\
14 & 4.960740 & 5.765951 & 5.880741 & 5.917820 & 5.943405 & 5.961695 & 5.974905 \\
15 & 5.015523 & 5.782492 & 5.890006 & 5.927062 & 5.952478 & 5.970624 & 5.984018 \\
16 & 5.064366 & 5.796996 & 5.898530 & 5.935471 & 5.960697 & 5.978779 & 5.992370 \\
17 & 5.108219 & 5.809869 & 5.906409 & 5.943178 & 5.968227 & 5.986299 & 6.000087 \\
18 & 5.147840 & 5.821408 & 5.913723 & 5.950291 & 5.975188 & 5.993286 & 6.007259 \\
19 & 5.183840 & 5.831843 & 5.920540 & 5.956897 & 5.981668 & 5.999814 & 6.013958 \\
20 & 5.216716 & 5.841350 & 5.926919 & 5.963065 & 5.987737 & 6.005941 & 6.020238 \\
21 & 5.246876 & 5.850070 & 5.932909 & 5.968852 & 5.993446 & 6.011714 & 6.026148 \\
22 & 5.274659 & 5.858114 & 5.938554 & 5.974304 & 5.998838 & 6.017172 & 6.031727 \\
\hline
\end{tabular}


TABLE 3. (Continued)

\begin{tabular}{|r|ccccccc|}
\hline$n$ & $k=7$ & 8 & 9 & 10 & 11 & 12 & 13 \\
\hline 8 & 5.875573 & & & & & & \\
9 & 5.915217 & 5.920173 & & & & & \\
10 & 5.934839 & 5.939744 & 5.941919 & & & & \\
11 & 5.949462 & 5.954945 & 5.958323 & 5.959963 & & & \\
12 & 5.962695 & 5.969311 & 5.974100 & 5.977255 & 5.978827 & & \\
13 & 5.974480 & 5.981845 & 5.987416 & 5.991411 & 5.993985 & 5.995248 & \\
14 & 5.984961 & 5.992822 & 5.998920 & 6.003522 & 6.006824 & 6.008964 & 6.010019 \\
15 & 5.994433 & 6.002722 & 6.009322 & 6.014523 & 6.018523 & 6.021448 & 6.023369 \\
16 & 6.003108 & 6.011782 & 6.018828 & 6.024532 & 6.029081 & 6.032600 & 6.035174 \\
17 & 6.011110 & 6.020114 & 6.027520 & 6.033604 & 6.038552 & 6042499 & 6.045544 \\
18 & 6.018531 & 6.027806 & 6.035498 & 6.041881 & 6.047148 & 6.051446 & 6.054887 \\
19 & 6.025440 & 6.034941 & 6.042870 & 6.049504 & 6.055048 & 6.059657 & 6.063446 \\
20 & 6.031902 & 6.041593 & 6.049724 & 6.056578 & 6.062365 & 6.067243 & 6.071328 \\
21 & 6.037967 & 6.047824 & 6.056130 & 6.063178 & 6.069177 & 6.074286 & 6.078621 \\
22 & 6.043681 & 6.053682 & 6.062143 & 6.069359 & 6.075540 & 6.080842 & 6.085381 \\
\hline
\end{tabular}

\begin{tabular}{|c|cccccccc|}
\hline$n$ & $k=14$ & 15 & .16 & 17 & 18 & 19 & 20 & 21 \\
\hline 15 & 6.024322 & & & & & & & \\
16 & 6.036860 & 6.037696 & & & & & & \\
17 & 6.047766 & 6.049221 & 6.049941 & & & & & \\
18 & 6.057557 & 6.059515 & 6.060802 & 6.061440 & & & & \\
19 & 6.066503 & 6.068889 & 6.070647 & 6.071805 & 6072381 & & & \\
20 & 6.074706 & 6.077441 & 6.079579 & 6.081155 & 6.082194 & 6.082194 & 6.082710 & \\
21 & 6.082267 & 6.085291 & 6.087744 & 6.089665 & 6.091083 & 6.092019 & 6.092484 & \\
22 & 6.089244 & 6.092500 & 6.095204 & 6.097398 & 6.099117 & 6.100385 & 6.101222 & 6.101638 \\
\hline
\end{tabular}

With $a_{2}, a_{3}, a_{4}, \ldots$ set to zero we have the ratio method, while relaxing this constraint gives the ratio method with Neville table extrapolation, in which subsequent columns of the table take into account successively higher terms in the polynomial in $n^{-1}$ above. The Neville table applied to the ratios of the $q_{\max }$ series is shown in Table 3, where only the first 24 ratios are extrapolated, as the lack of precision in the higher coefficients renders them useless for the higher-order columns. It can be seen that all columns are monotonically increasing, and reading from left to right, all rows are monotonically increasing. The remarkable behaviour immediately suggests that the singularity is not of the assumed form, that is, not a conventional algebraic singularity.

This is a most unusual situation, and before accepting this conclusion we tried a variety of other techniques. Firstly, we applied the method of Pade approximants to the logarithmic derivative of the series, calculating both diagonal and off-diagonal approximants. The nearest singularity to the origin at each order, as estimated by the nearest non-defective pole, was substantially beyond $M_{*}^{2}$, as shown in Figure 1. There we plot the nearest pole against $1 /(N+D)$ where $N$ 


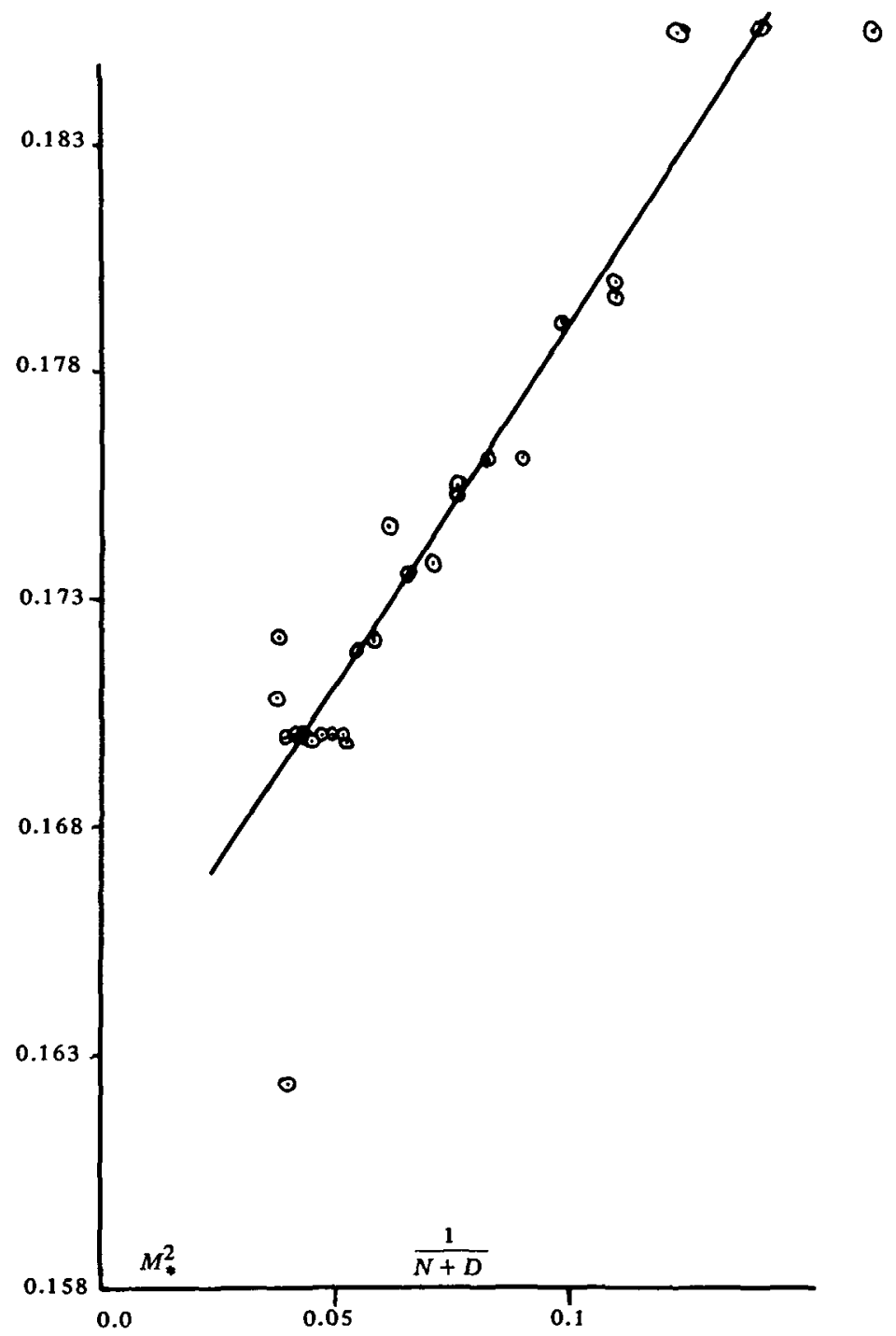

Figure I. Plot of nearest non-defective pole of logarithmic derivative Padé approximants to $q_{\max }\left(M^{2}\right) / U$ series versus $1 /(N+D)$. Diagonal and off-diagonal approximants used.

and $D$ are the degrees of the numerator and denominator polynomials respectively. The essentially linear plot is suggestive of an extrapolated value beyond $M_{*}^{2}$, and hence suggests that $M_{c}>M_{*}$. Nevertheless, it should be recalled that the Padé method is intrinsically unsatisfactory for dealing directly with cusp-like 
singularities, that is, singularities of the form

$$
f(x) \sim A(x)+B(x)(1-\mu x)^{\alpha},
$$

with $\alpha>0$ (except for special cases, such as $0<\alpha<1$ and $A(1 / \mu)=0$ ).

Such singularities can be analysed by the recurrence-relation method, introduced by Guttmann and Joyce [7, 12]. In this method, the available series coefficients are fitted to the expansion of a $K$ th-order differential equation of the form

$$
\sum_{i=0}^{K} P_{i}(x) f^{(i)}(x)=Q(x)
$$

where $P_{t}(x)$ are polynomials, and $Q(x)$ is identically zero or a polynomial. Here, $f^{(i)}(x)$ denotes the $i$ th derivative of $f$. This equation gives rise to solutions with quite a rich variety of singularity types [7]. In particular, solutions of the form (13) are contained, as are confluences of conventional algebraic singularities with logarithmic factors. The application of this method confirmed our earlier conclusion that the singularity is not of the conventional algebraic type, as the estimates of $M_{c}^{2}$ were quite erratic.

Experience with problems in critical phenomena suggested two further possible singularity types. One of these is a confluent singularity structure, of the form

$$
f(x) \sim A(x)(1-\mu x)^{\alpha}+B(x)(1-\mu x)^{\beta}+C(x)(1-\mu x)^{\gamma}+\cdots
$$

where $\alpha, \beta, \gamma, \ldots$ are not separated by integers. The other is an exponential singularity, of the form

$$
f(x) \sim A(x)+B(x) \exp \left[c /(1-\mu x)^{\alpha}\right], \quad \alpha>0 .
$$

Singularities of the confluent type can be identified by a suitable modification of the recurrence-relation method, as shown by Rehr et al.[19]. Attempts at such an identification were unsuccessful. An alternative method devised by Baker and Hunter [1] involves transforming the original power series, using a highly non-linear transformation, in such a way that the reciprocals of the exponents $\alpha, \beta, \gamma, \ldots$ appear as poles of Padé approximants. The method does however require accurate knowledge of the position of the singularity, $x=1 / \mu$. Choosing a range of values for $1 / \mu$, including $M_{*}$ and a posteriori $M_{c}$, no remotely consistent estimates of any exponents were obtained. We therefore discounted the possibility of a singularity structure of a confluent type.

The exponential singularity is more subtle than the conventional algebraic singularity, as its nature depends crucially on the sign of the constant $c$ in equation (16). If $c>0$, then as $x \rightarrow 1 / \mu-$, the singularity diverges more strongly than any simple power. If $c<0$ however, then as $x \rightarrow 1 / \mu-$, the singular part 
vanishes faster than any power, and the function is, generally speaking, numerically indistinguishable from its analytic part $A(x)$.

Nevertheless, we found that certain test series with this singularity structure display some, but not all, of the features observed in the $q_{\max }$ series. In particular, the function

$$
g(x)=\exp (-x / 4)+\exp \left[-0.67 /(1-x)^{1 / 4}\right]=\sum a_{n} x^{n}
$$

qualitatively reproduces the monotonic trend observed in both the rows and columns of the Neville table. One fundamental difference however is that Padé approximants to $g(x)$ generally have their closest pole nearer to the origin than the radius of convergence of the series, $x=1$, while as mentioned previously, the nearest pole of Padé approximants to the $q_{\max }$ series is well beyond $M_{*}$. This difference is fundamental, and suggests that the singularity is not of exponential type. This conclusion is strengthened by an alternative analysis, in which we take the logarithmic derivative of the logarithmic derivative of the original series, and then study the behaviour of the Padé approximants. As shown by Guttmann [6], this procedure will, under certain circumstances, convert singularities of the form (16) into meromorphic functions. Once again we found no support for an essential singularity of this type.

Returning to the Neville table, Table 3, the observed monotonic behaviour, which also displays itself as non-removable curvature in the ratio plots, is reminiscent of the situation in numerical analysis wherein one attempts to fit a function, for example $\sqrt{x}$, by a polynomial. Such an attempt will never be satisfactory, due to the essentially non-polynomial nature of the function. Indeed, the ratios, shown in the first column of Table 3 appear to be behaving like $r_{n} \sim \mu\left(1-c / n^{\alpha}\right)$, with $\alpha<1$. The underlying singularity structure suggested by such behaviour is not known generally, though it can be found for the special case $\alpha=1 / m$, where $m$ is an integer. However, very recently Barber and Hamer [2] have developed an extrapolation technique for sequences of precisely this type. Their method relies on the judicious application of a one-parameter family of non-linear transformations introduced by Vanden Broeck and Schwartz [26]. The transformations are defined by the equations

$$
\begin{gathered}
\varepsilon_{n}^{(m)}=\alpha \varepsilon_{n}^{(m-1)}+1 /\left(f_{n+1}^{(m)}-f_{n}^{(m)}\right), \\
f_{n}^{(m+1)}=f_{n}^{(m)}+1 /\left(\varepsilon_{n}^{(m)}-\varepsilon_{n-1}^{(m)}\right)
\end{gathered}
$$

where $\alpha$ is an arbitrary parameter, $\varepsilon_{n}^{(1)} \equiv 0$, and the $f_{n}^{(0)}, n \in[1, N]$, are the original sequence coefficients. As shown by Vanden Broeck and Schwartz [26], setting $\alpha=0$ recovers the Shanks transformation, already used to estimate $M_{*}$, while $\alpha=1$ corresponds to Wynn's $\varepsilon$-algorithm [29] for generating diagonal Padé approximants, though in that case $f_{n}^{(0)}$ is not the $n$th coefficient but the $n$th partial 
sum. Barber and Hamer have proved the following relevant theorem:

THEOREM. Let $\left\{f_{n}\right\}$ approach its limit $f_{\infty}$ as

$$
f_{n}-f_{\infty}=A n^{-\lambda}\left[1+b n^{-\Delta}+o\left(n^{-\Delta}\right)\right], \quad n \rightarrow \infty .
$$

Then

$$
f_{n}^{(2)}(\alpha=-1)-f_{\infty}=A n^{-\lambda} \rho_{n}
$$

where

$$
\rho_{n}= \begin{cases}o\left(n^{-1}\right), & \Delta \geqslant 1, \\ O\left(n^{-\Delta}\right), & \Delta<1 \quad \text { as } n \rightarrow \infty .\end{cases}
$$

Thus $f_{n}^{(2)}$ should converge more rapidly than $f_{n}^{(0)}$. The theorem also suggests that repeated application of the transformation with $\alpha=-1$ will accelerate convergence further. However in so doing the parameter $\alpha$ in $\varepsilon_{n}^{(2)}$ must be reset to zero to nullify the contribution from $\varepsilon_{n}^{(1)}$. Therefore successive transformations are carried out with $\alpha=-1$, then $\alpha=0$, and so on. The sequence of transformations is therefore given by

$$
\begin{gathered}
f_{n}^{(m+1)}=f_{n}^{(m)}+1 /\left[\varepsilon_{n}^{(m)}-\varepsilon_{n-1}^{(m)}\right], \\
\varepsilon_{n}^{(m)}=\alpha_{m} \varepsilon_{n}^{(m-1)}+1 /\left[f_{n+1}^{(m)}-f_{n}^{(m)}\right]
\end{gathered}
$$

where

$$
\alpha_{m}=\left\{\begin{array}{ll}
0 & \text { if } m \text { is even } \\
-1 & \text { if } m \text { is odd }
\end{array}\right\} .
$$

Application of this method is generally limited by one or both of the following features. At each iterative cycle from $f_{n}^{(2 m)}$ to $f_{n}^{(2 m+2)}$ four terms of the sequence are lost. Secondly, the number of significant digits lost at each iteration can be quite large. Nevertheless, applying this sequence of transformations to the ratios of successive coefficients of the $q_{\max }$ series, we obtained the sequences $f_{n}^{(2)}$ to $f_{n}^{(10)}$ before running out of terms. In deriving this table, we used only the first 24 coefficients, which were available in quadruple precision. The remaining coefficients were known to only a few significant figures (see Table 1). In order to guard against loss of accuracy, all calculations were performed in quadruple precision on a DEC VAX 11/780 computer. The results are shown in Table 4, wherein it can be seen that the sequences $f_{n}^{(6)}$ to $f_{n}^{(10)}$ display good convergence, and allow us to conclude that

$$
M_{c}^{-2}=6.16746391 \pm 0.00000015 \text {. }
$$

We also used this method to extrapolate the sequence of estimates of $M_{*}$. These results are shown in Table 5, wherein it is seen that convergence is much more 

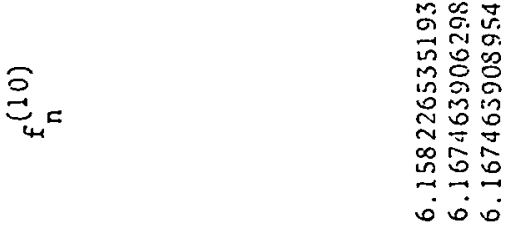

告

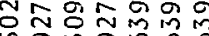

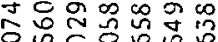

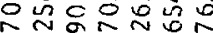

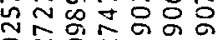
in

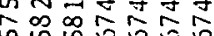

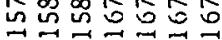
ம் $\dot{0} \dot{0} \dot{0} \dot{0}$

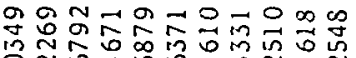

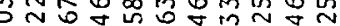
ô

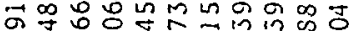

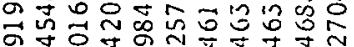

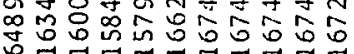
ம் $\dot{0} \dot{0} \dot{0} \dot{0} \dot{0} \dot{0} \dot{0}$

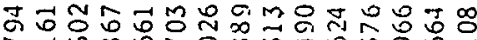

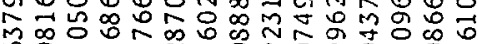

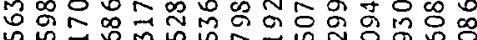

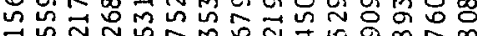

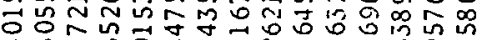

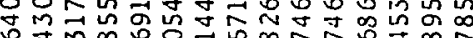

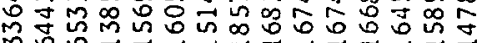
ம் $\dot{0} \dot{0} \dot{0} \dot{0} \dot{0} \dot{0} \dot{0} \dot{0}$

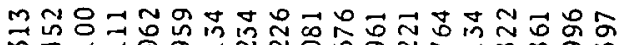

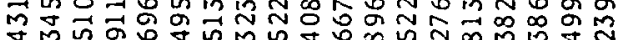

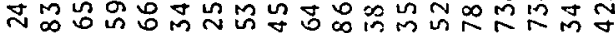

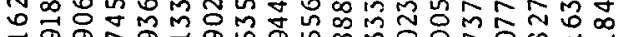

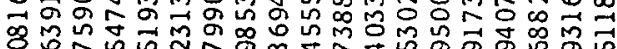

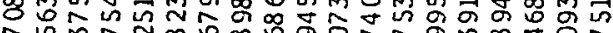

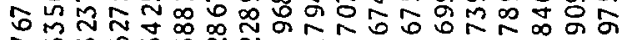

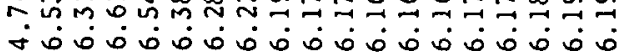

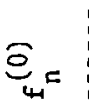

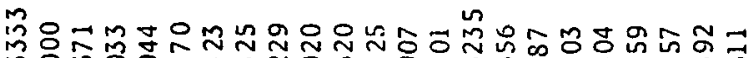

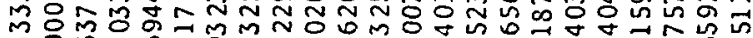
mㅇํㅇ

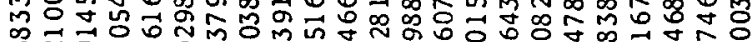
品 O N

- $\rightarrow$ m 
$\Xi_{4-1}^{\Xi}$

N

政

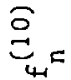

$\overbrace{4}^{\infty}=$

$\underbrace{0}_{+1}$

$\overbrace{4=}^{\Xi}$
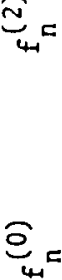

c

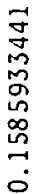

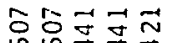

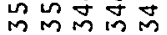

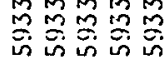

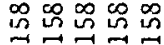

சं

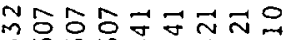

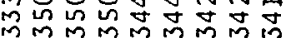

लm

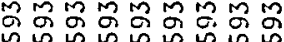

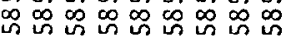

$\neg \neg \neg \neg \neg \neg \neg$

ல் $\dot{0} 0 \dot{0} 0 \dot{0}$

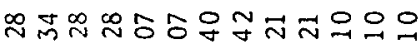
m $m$ in M $M M M M M M M M$

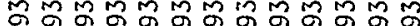
ํํำ

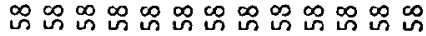

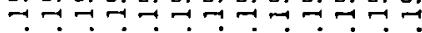
வ்0ல் $\dot{0} 0 \dot{0} 0 \dot{0}$

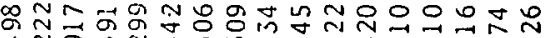

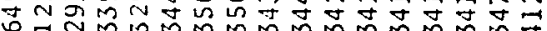

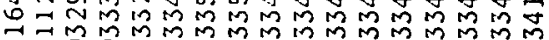
穴

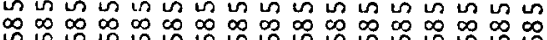

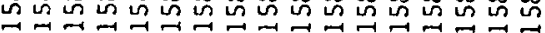
:0ं0்

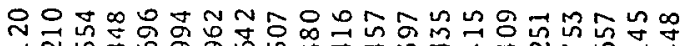

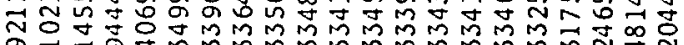
\%

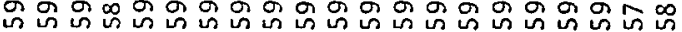

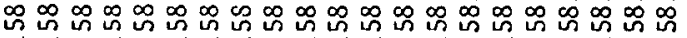
ப்

ㅎํํㅇำ ผ ஸि

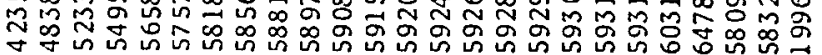

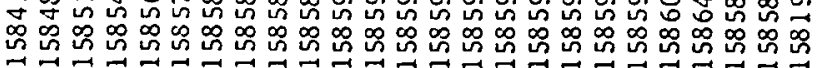

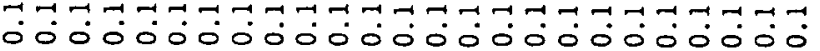

mo

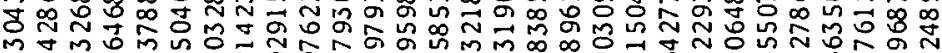

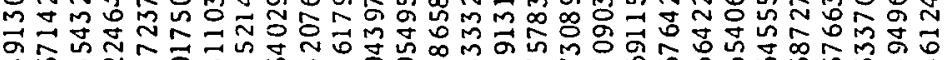
क人

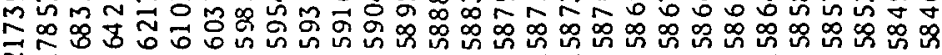
กี ○ं்

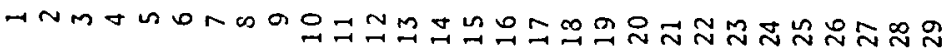


rapid than for the corresponding Shank's table, Table 2, and allows us to make the estimate $M_{*}=0.39823780 \pm 0.00000001$.

We have been able to estimate $M_{*}$ by a variety of methods, of which two are shown here, and all methods give values of $M_{*}$ in agreement with that quoted above, though in most cases with lower precision. For the estimate of $M_{c}$ however, we base our estimate only on one comparatively-untried method. Additionally, we are unable to determine the nature of the singularity at $M=M_{c}$, though we have excluded a number of the more conventional possibilities. Accordingly, while the sequence of estimates of $M_{c}$ appear to display a level of precision consistent with the confidence limits quoted, the possibility of spurious convergence must be admitted.

\section{Discussion and conclusion}

Our primary conclusion is that $M_{c}>M_{*}$, by some $1.1 \%$ with values of $M_{c}$ and $M_{*}$ as quoted earlier. Hence the transonic controversy is resolved in that it has been shown that a circular "airfoil" can indeed have a continuous range of smooth, shock-free potential flows above the critical Mach-number. With the long-standing transonic controversy resolved, the practical question that should be answered next is whether, in the continuous range of shock-free flows above the critical Mach number, the solution is stable.

Our result gains some support from two independent sources. Seebass (private communication) has calculated flows past a circular cylinder numerically, using Jameson's-FLO 6 program. His results are that at $M=0.41$, which is greater than $M_{*}$, there is no hint of a shock wave numerically, while at $M=0.43$ the shock wave is clear. This suggests a region of shock-free flow above $M_{*}$. The disappointing aspect of this result is that the value, $M=0.41$, found to be shock-free, is also greater than $M_{c}=0.4026 \ldots$, and so cannot be shock-free. Hence Seebass' result cannot be regarded as totally convincing. Presumably it arises because the nature of the singularity at $M_{c}$ is such that it does not manifest itself clearly in the pressure distribution and related quantities calculated by Seebass until the shock wave is well developed.

The second source that provides support for our result stems from a detailed study of the nature of the $q_{\max } / U$ series at $M^{2}=M_{*}^{2}$. By forming diagonal and off-diagonal Padé approximants to $q_{\max } / U$ and evaluating these at $M_{*}^{2}$, we find the approximants to be well converged, as shown in Table 6 , permitting us to make the estimate

$$
q_{\max }\left(M_{*}^{2}\right) / U=2.328346
$$


TABLE 6. Diagonal and off-diagonal Padé approximants to $q_{\max }\left(M^{2}\right) / U$ series, evaluated at $M^{2}=M_{*}^{2}=0.15859334$.

\begin{tabular}{|r|c|c|c|}
\hline$N$ & {$[N-1 / N]$} & {$[N / N]$} & {$[N+1 / N]$} \\
\hline 5 & 2.327897 & 2.328103 & 2.328198 \\
6 & 2.328198 & 2.328263 & 2.328295 \\
7 & 2.328295 & 2.328317 & 2.328328 \\
8 & $2.328328 \overline{8}$ & 2.328336 & 2.328340 \\
9 & 2.328340 & 2.328342 & 2.328344 \\
10 & 2.328344 & 2.328345 & 2.328346 \\
11 & 2.328346 & 2.328346 & 2.328346 \\
12 & 2.328346 & 2.328346 & 2.328346 \\
13 & 2.328346 & 2.328346 & 2.328346 \\
14 & 2.328346 & 2.328346 & \\
\hline
\end{tabular}

By subtracting this value of $q_{\max }\left(M_{*}^{2}\right) / U$ from the original series-that is, altering the first coefficient to -0.328346 - and investigating the remaining series by forming Pade approximants to the logarithmic derivative, we find the behaviour of the poles and zeros to imply, absolutely clearly, that the remaining series behaves like const. $\times\left(M^{2}-M_{*}^{2}\right)$. Evaluating the constant, and removing the contribution of that term from the series gives a new series which, upon similar investigation, is found to be dominated by a term of the form const. $X$ $\left(M^{2}-M_{*}^{2}\right)^{2}$. That is, we find

$$
q_{\max }\left(M^{2}\right) / U=A_{0}+A_{1}\left(M^{2}-M_{*}^{2}\right)+A_{2}\left(M^{2}-M_{*}^{2}\right)^{2}+\cdots,
$$

which strongly implies that the series is analytic at $M_{*}^{2}$. This is, of course, consistent with our conclusion that $M_{c}>M_{*}$.

While this calculation lends some support to our conclusions, it is not unequivocal for a similar reason that Seebass' result is not completely convincing. If the singularity is sufficiently weak, the process we have just described would not detect it. As an example, consider the function $f(x)=e^{-0.67 /(1-x)^{1 / 4}}+e^{-x}$. Given the first 25 coefficients in the series expansion of $f$, the Padé approximant study we have just discussed implies that $f$ is analytic at $x=1$, a result which is clearly false. This misleading result is, of course, attributable to the nature of the singularity, which is numerically imperceptible near $x=1$.

Nevertheless, both the calculation of Seebass and the study of the nature of the singularity at the critical Mach-number are valuable in that they provide checks on our result in the sense of necessity rather than sufficiency.

There still remain two unanswered questions whose resolution would considerably enhance our understanding of this problem and indeed our confidence in our major conclusion. The first relates to the nature of the singularity. We have shown 
fairly convincingly that it is not of the conventional algebraic or algebraico-logarithmic type, nor is it likely to be a confluent assemblage of such singularities. Further, it is unlikely to be of exponential type, though in this case our results are less convincing. Indeed, a recent calculation by Bollmann [3] suggests that this possibility should be studied more carefully. Bollmann has studied subsonic flow along a sinusoidal wall in the transonic small-disturbance approximation. $\mathrm{He}$ generates 36 terms in the series expansion for a variety of flow quantities, and finds that the series is a Stieltjes series. That is, it can be written as

$$
f(z)=\sum_{n \geqslant 0} c_{n} z^{n}=\int_{\alpha}^{\beta} \frac{\rho(x) d x}{1-x z} ; \quad c_{n}=\int_{\alpha}^{\beta} x^{n} \rho(x) d x .
$$

Bollmann claims that an analysis of the moment-generating function $\rho(x)$ suggests an exponential singularity of the type we have previously discussed, and that $\beta=-\alpha=1 / z$ so that the series converge only up to the critical Mach-number. The series for our problem is not a Stieltjes series, and so Bollmann's procedure cannot be applied. There is also some doubt whether Bollmann's series satisfy all necessary conditions for a Stieltjes series.

The second question that arises relates to the generality of our result, that $M_{c}>M_{*}$. Given that it applies to a circle, it is quite clear that it will also apply to any ellipse of low eccentricity, and it is reasonable to assume that it applies to most convex, analytic airfoils. But what of non-analytic airfoils? Nocilla's [16] numerical results on biconvex airfoils suggest that analyticity of the airfoil is not a necessary condition. In a study of a biconvex airfoil with a $45^{\circ}$ vertex angle he finds a critical Mach number of 0.531 , but smooth flows seem to be present at Mach numbers in excess of 0.55 . One must nevertheless remain concerned at the possibility of a weak singularity not being numerically visible until the shock wave is well developed, as seems to be the case with Seebass' calculation.

\section{Acknowledgements}

We wish to thank L. Schwartz and R. Seebass for their helpful suggestions, and M. N. Barber and G. Bollmann for supplying a preprint of their work prior to publication. We also wish to thank J. B. Keller for useful discussions, and E. Drummond and M. N. Barber for comments on the method of analysis.

This work was partially supported by the U. S. Air Force Office of Scientific Research under Grant AFOSR 74-2649 and by the National Science Foundation under Grant ENG-7824412. 


\section{References}

[1] G, A. Baker Jr. and D. L. Hunter, "Methods of series analysis II. Generalized and extended methods with application to the Ising model", Phys. Rev. B 7 (1973), 3377-3392.

[2] M. N. Barber and C. J. Hamer, "Extrapolation of sequences using a generalized epsilon algorithm", J. Austral. Math. Soc. Ser. B 23 (1982), 229-240.

[3] G. Bollmann, "Potential flow along a wavy wall and the transonic controversy", submitted to $J$. Engrg. Math. (!982).

[4] F. I. Frankl and M. V. Keldysh, "The exterior Neumann problem for nonlinear elliptic differential equations with application to the theory of a wing in a compressible gas" (Russian), Izv. Akad. Nauk SSSR 12 (1934), 561-601.

[5] D. S. Gaunt and A. J. Guttmann, "Asymptotic analysis of coefficients", in Phase Transitıons and Critical Phenomena, Vol. 3 (eds. C. Domb and M. S. Green), (Academic Press, New York, 1974).

[6] A. J. Guttmann, "The critical behaviour of two-dimensional isotropic spin systems", J. Phys. A 11 (1978), 545-553.

[7] A. J. Guttmann and G. S. Joyce, "On a new method of series analysis in lattice statıstics", $J$. Phys. A 5 (1972), 181-84.

[8] G. H. Hoffman, "Computer extension of some perturbation series in fluid mechanics", (Stanford University, Department of Aero. and Astro., Ph.D. thesis, 1970; Uni. Microfilms order No. $71-12,920$ ).

[9] G. H. Hoffman, "Extension of perturbation series by computer: Symmetric subsonic potential flow past a circle", J. Mécanıque 13 (1974), 433-447.

[10] 1. Imai, "On the flow of a compressible fluid past a circular cylinder I and II", Phys. Math. Soc. Japan Proc. Ser. 320 (1938), 636-645 and 23 (1941), 180-193.

[11] O. Janzen, "Beitrag zu einer Theorie der stationären Strömung kompressibler Flüssigkeiten, Phys. Zeits. 14 (1913), 639-43.

[12] G. S. Joyce and A. J. Guttmann, "A new method of series analysis" in Padé approximants and their applications (ed. P. R. Graves-Morris), (Academic Press, London, 1973), 163-167.

[13] M. J. Lighthill, "Higher approximations", in General theory of high speed aerodynamics (ed. W. R. Sears), (Princeton Univ. Press, Princeton, 1954).

[14] R. E. Melnick and D. C. Ives, "Subcritical flows over two dimensional airfoils by a multistrip method of integral relations", Proc. 2nd Internat. Conf. on Numer. Methods in Fluid Dyn., Berkeley, CA, 1970, (Springer-Verlag, Berlin, 1971), 243-251.

[15] C. Morawetz, "On the non-existence of continuous transonic flow past profiles I, II and III", Comm. Pure Appl. Math. 9 (1956), 45-68, 10 (1957), 107-131, and 11 (1958), 129-144.

[16] S. Nocilla, G. Geymonat and B. Gabutti, "The direct problem of the transonic airfoils on the hodograph", Symposium Transsonicum II (eds. K. Oswatitsch and D. Rues), (Springer-Verlag, 1976), 134-141.

[17] K. Oswatitsch, Gas dynamics (Academic Press, New York, 1956).

[18] Lord Rayleigh, "On the flow of compressible fluid past an obstacle", Phtl. Mag. 32 (1916), l-6.

[19] J. Rehr, G. S. Joyce and A. J. Guttmann, "A recurrence technique for confluent singularity analysis of power series", J. Phys. A 13 (1980), 1587-1602.

[20] R. Sauer, Introduction to theoretical gas dynamics (Edwards, Ann Arbor, 1947).

[21] R. Sauer, Einführung in die theoretische Gasdynamik (Springer, Berlin, 1960).

[22] D. Shanks, "Non-linear transformations of divergent and slowly convergent sequences", $J$. Math. and Phys. 34 (1955), 1-42.

[23] A. H. Shapiro, The dynamics and thermodynamics of compressible fluid flow (Ronald, New York, 1954).

[24] T. Simasaki, "On the flow of a compressible fluid past a circular cylinder, I", Bull. Naniwa Univ. Ser. A 3 (1955), 21-30. 
[25] G. I. Taylor and C. F. Sharman, "A mechanical method for solving problems of flow in compressible fluids", Proc. Roy. Soc. London Ser. A 121 (1928), 194-217.

[26] J. M. Vanden Broeck and L. W. Schwartz, "A one-parameter family of sequence transformations", SIAM J. Math. Anal. 10 (1979), 658-666.

[27] M. Van Dyke and A. J. Guttmann, "Computer extension of the $M^{2}$ expansion for a circle", Bull. Amer. Phys. Soc. 23 (1978), 996.

[28] G. N. Ward, "Approximate methods", in Modern Developments in Fluid Mechantcs, High Speed Flow, Vol. 1 (ed. L. Howarth), (Oxford Univ. Press, London, 1953).

[29] P. Wynn, "Upon systems of recursions which obtain among the quotients of the Pade table", Numer. Math. 8 (1966), 264-269. 